\title{
Effect of tillage system and straw management on organic matter dynamics
}

\author{
Samarendra HAZARIKA ${ }^{1 *}$, Robert PARKINSON ${ }^{2 * *}$, Roland BOL $^{3}$, Liz DIXON $^{3}$, Peter RUSSELL $^{2}$, Sarah DonOVAN $^{2}$, \\ Debbie ALLEN ${ }^{4}$ \\ ${ }^{1}$ Dept. of Agricultural Engineering, Assam Agricultural University, Jorhat-785 013, Assam, India \\ ${ }^{2}$ School of Biological Sciences, University of Plymouth, Drake Circus, Plymouth, PL4 8AA, UK \\ ${ }^{3}$ Soil and Water team, Cross Institute Programme for Sustainable Soil Function, North Wyke Research, Okehampton, Devon EX20 2SB, UK \\ ${ }^{4}$ Plant Genetics and Breeding, Institute of Biological, Environmental and Rural Sciences, Aberystwyth University, SY23 3EB, UK
}

(Accepted 6 July 2009)

\begin{abstract}
The choice of cultivation system in arable agriculture exerts a strong influence not only on soil health and crop productivity but also on the wider environment. Conservation tillage using non-inversion methods conserves soil carbon, reduces erosion risk and enhances soil quality. In addition, conservation tillage has been shown to sequester more carbon within the soil than inversion tillage, reducing carbon dioxide losses to the atmosphere. Stable, well structured topsoils that develop following long-term conservation tillage lead to more energy efficient systems due to the reduced power requirements for cultivation. Long-term experiments, e.g. more than 20 years, that confirm the impact of conservation tillage over an extended period are not common. Here we evaluate the impact of different tillage methods and winter wheat straw management, either incorporated or removed, on organic matter turnover and soil quality indicators. No-till, chisel and mouldboard ploughing was carried out for 23 years on a silty clay loam soil in South West England that was not considered suitable for non-inversion tillage due to weak soil structure. In order to assess the effect of contrasting cultivation and straw disposal method on soil carbon dynamics, a range of assays were conducted, including water extractable organic carbon, hot water extractable carbohydrate, microbial biomass carbon, activity of $\beta$-glucosidase and acid phosphatase enzymes, $\mathrm{C}$ sequestration and the natural abundance of ${ }^{13} \mathrm{C}$. Our results show that the soil organic carbon concentration in the topsoil was greater under no-till than mouldboard ploughing, while a reverse trend was observed in the lower depths. A 14-17\% increase in soil organic carbon was observed in the topsoil for chisel plough and no-till treatments compared to mouldboard ploughing. Water extractable organic carbon was found to constitute only $1-7 \%$ of the microbial biomass carbon. Hot water extractable carbohydrate was one of the most sensitive indicators of soil quality and had a significant a negative correlation with bulk density and positive correlation with soil organic carbon microbial biomass carbon $\beta$-glucosidase and acid phosphatase. The choice of cultivation method exerted a major control on microbial and carbon dynamics. No-till and chisel ploughing maintained carbon in the soil surface horizons, which will benefit the stability of this weakly structured soil, but mouldboard ploughing distributed carbon more uniformly throughout the soil profile, particularly when straw was incorporated, hence leading to the retention of more carbon in the soil profile.
\end{abstract}

soil quality / carbon sequestration / tillage / straw management / winter wheat

\section{INTRODUCTION}

The choice of tillage system allows farmers to optimise the soil environment for crop plants and achieve important environmental benefits. The adoption of conservation tillage methods such as minimum cultivation and residue incorporation

* Present address: Central Horticultural Experiment Station (CHES), Indian Institute of Horticultural Research, Chettalli-571 248, North Kodagu, Karnataka, India.

**Corresponding author: rparkinson@plym.ac.uk can reduce carbon emissions and enhance soil structural stability, thus reducing erosion risk (Zuazo and Pleguezuelo, 2008). Only by understanding the complexity of organic matter transformations under contrasting tillage can the full environmental and agronomic benefit be determined. The potential negative effects of conventional or inversion tillage (ploughing) in arable cropping systems, such as the loss of organic matter and structure degradation, can be countered by the use of conservation tillage practices to improve soil quality (Rasmussen and Collins, 1991; Roberto et al., 1995; Scopel et al., 2005; Lal, 2008; He et al., 2009). The combined effect of non-inversion 
tillage and straw incorporation on the accumulation of soil organic carbon was reported to be greater than the effect of either tillage reduction or straw incorporation alone (Kushwaha et al., 2001). West and Post (2002) analyzed data from longterm studies across the world and reported an average $\mathrm{C}$ gain of $0.57 \mathrm{Mg} \mathrm{C} \mathrm{ha-} \mathrm{yr}^{-1}$ following a change from inversion tillage to no-tillage systems. No-tillage systems not only conserve soil organic matter, but can reduce greenhouse gas emissions (Smith et al., 1998).

Observed changes in other biological quality indicators due to straw incorporation following adoption of no-till systems include enhanced microbial activity and biomass (Ahl et al., 1998; Montemurro et al., 2007). In general, microbial biomass and microbial processes in the surface soil under reduced tillage are significantly greater than those in ploughed soils. Hot water extractable carbohydrate and water extractable carbon, being a highly labile component of soil organic matter, are sensitive to soil management practices (Doran and Parkins, 1994) and therefore can be used as a sensitive indicator of soil quality. Phosphatase, $\beta$-glucosidase and urease activity in soil are most frequently used among the specific biochemical soil quality parameters and appropriately, represents the $\mathrm{C}$, $\mathrm{N}$ and P cycles (Gil-Sotres et al., 2005). Deng and Tabatabai (1997) investigated the effect of tillage and straw management on enzyme activities in soils, and found that most of the enzymes studied were present in significantly greater concentrations in no-till than in other tillage systems.

Long term field experiments represent a valuable source of information on the impact of agronomy on the carbon sequestration, turnover of soil organic matter, and change in soil quality indicators over time (Lichtfouse, 1997; Carter, 2005; Lichtfouse et al., 2005; Benbi and Brar, 2009). In this paper we report the effect of contrasting tillage and straw management practices on $\mathrm{C}$ sequestration, organic matter turnover and soil quality indicators from a long-term (23 year) winter wheat cultivation trial in South West England.

\section{MATERIALS AND METHODS}

\subsection{Experimental design and tillage treatments}

A long term field experiment was established in 1982 at the University of Plymouth Farm, Seale-Hayne, Devon, South West England (National Grid Reference SX 823722) to assess the impact of contrasting tillage on the agronomy of winter wheat (Triticum aestivum L.). The long-term average rainfall for the site is $975 \mathrm{~mm}$ per annum. The experiment was designed to investigate soil and crop responses to three tillage treatments: no-till, chisel plough (minimum tillage) and mouldboard plough (conventional tillage). The experimental site was divided into three blocks within which each treatment was replicated three times. Treatments were allocated randomly within each block. Each treatment plot measured $40 \times 5 \mathrm{~m}$. Initially, all straw was baled and removed from the plots prior to stubble burning. However, following the ban on straw burning in England in 1993, each plot was subdivided
Table I. Summary of tillage and straw disposal treatments used in this experiment. The target tillage depth was as follows: mouldboard plough 150-180 mm; chisel plough 120-150 mm; heavy disc 80$100 \mathrm{~mm}$; spring tine and light disc 50-80 mm. All plots were power harrowed at $50-60 \mathrm{~mm}$ and rolled to incorporate stubble prior to primary tillage.

\begin{tabular}{lcc}
\hline $\begin{array}{l}\text { Primary } \\
\text { tillage }\end{array}$ & $\begin{array}{c}\text { Straw disposal } \\
\text { Method }\end{array}$ & $\begin{array}{c}\text { Secondary } \\
\text { tillage }\end{array}$ \\
\hline $\begin{array}{l}\text { Mouldboard } \\
\text { plough }\end{array}$ & Chopped \& & Heavy disc, \\
Mouldboard & incorporated & light disc, spring tine \\
plough & Reavy disc, \\
Chisel & Chopped \& & light disc, spring tine \\
plough & incorporated & Heavy disc $\times 2$, \\
Chisel & light disc, spring tine \\
plough & Removed & Heavy disc $\times 2$, \\
No-till & light disc, spring tine \\
& Incopped \& & Light disc \\
No-till & Removed & Light disc \\
\hline
\end{tabular}

into two $20 \times 5 \mathrm{~m}$ plots, and an additional treatment superimposed on the original experiment, with straw either removed or incorporated. These straw treatments were allocated on a random basis. Hence since 1993 the experiment has consisted of six treatments, replicated three times. Full treatment details are given in Table I. Following primary and secondary cultivation all plots receive the same husbandry inputs appropriate to winter wheat production in South West England.

\subsection{Soil sampling and analysis}

The experimental plots are underlain by a dystric cambisol of the Denbigh Association, derived from Devonian slates and mudstones. The soil at the experimental site is a silt loam ( $0.08 \mathrm{~g} \mathrm{~g}^{-1}$ sand, $0.81 \mathrm{~g} \mathrm{~g}^{-1}$ silt and $0.11 \mathrm{~g} \mathrm{~g}^{-1}$ clay) and is classed as structurally weak (Findlay et al., 1984). Soil depth to in situ slate varies between $450-650 \mathrm{~mm}$. Soil samples were collected in November 2005. Four soil cores (volume $220 \mathrm{~cm}^{3}$ ) were randomly taken from each replicate at three depths, with the sample mid-point being 50,150 and $250 \mathrm{~mm}$. Throughout this paper the $50 \mathrm{~mm}$ soil depth is referred to as the topsoil. Samples were air-dried and ground to pass a $2 \mathrm{~mm}$ sieve prior to analysis.

Soil $\mathrm{pH}$ was measured in a 1:2.5 soil:water suspension. Soil organic carbon, total $\mathrm{N},{ }^{13} \mathrm{C}$ and ${ }^{15} \mathrm{~N}$ were determined by continuous flow-isotope ratio mass spectrometry, using automated $\mathrm{N}$ analysis mass spectrometry. Ground wheat flour was used as the working standard and analysed after every tenth sample, resulting in an analytical precision of $<0.1$ and $0.2 \%$ of $\delta^{13} \mathrm{C}$ and $\delta^{15} \mathrm{~N}$ units, respectively. Natural abundance of ${ }^{13} \mathrm{C}$ was expressed as $\delta^{13} \mathrm{C}_{\mathrm{VPDB}}$ which represents the ratio ${ }^{13} \mathrm{C}:{ }^{12} \mathrm{C}$ relative to the Vienna PDB standard. The $\delta^{13} \mathrm{C}$ values were defined as [atom $\%{ }^{13} \mathrm{C}_{\text {sample }}-$ atom $\%{ }^{13} \mathrm{C}_{\text {standard }} /$ atom $\%{ }^{13} \mathrm{C}_{\text {standard }}$ ] $\times$ 1000. As the soil samples were free of lime, the measured TOC solely comprised organic C. Soil organic carbon and total $\mathrm{N}$ in the $0-100$ and $100-200 \mathrm{~mm}$ depth layers was compared on an 
equivalent mass basis. This method eliminates the potentially erroneous comparisons that might result from calculating the $\mathrm{C}$ storage as the product of concentrations, bulk density and depth (Ellert and Bettany, 1995). The mass of the heaviest soil layer in the latter approach most susceptible to the influence of management is designated as the "equivalent" mass. In our case, the highest mass of soil in $50 \mathrm{~mm}$ depth was found to be under the no-till with straw removed treatment $\left(1.21 \mathrm{Mg} \mathrm{m}^{-3}\right)$ treatment while in case of $150 \mathrm{~mm}$ depth, it was under chisel plough straw removed treatment $\left(1.32 \mathrm{Mg} \mathrm{m}^{-3}\right)$. Soil organic carbon, total $\mathrm{N}$ and microbial biomass carbon stocks were calculated for the 0-200 $\mathrm{mm}$ soil depth using the procedure described by Ellert and Bettany (1995). Carbon stock was not calculated for the 200-300 mm depth, as this method requires bulk density data for the soil depth immediately below that for which the calculation is made, and this experiment bulk density data was not collected for the $350 \mathrm{~mm}$ depth.

The concentration of microbial biomass carbon in soil was determined following a modified fumigation-extraction method (Gregorich et al., 2000). The microbial biomass carbon was calculated as the difference in soluble organic $\mathrm{C}$ between the fumigated and unfumigated extracts, divided by 0.45 , as recommended by Wu et al. (1990). The fraction of the SOC extracted with water in unfumigated sample was classified as water extractable organic carbon. Hot water extractable carbohydrate was obtained by incubating field moist soil samples with distilled water at $80{ }^{\circ} \mathrm{C}$ for $16 \mathrm{~h}$. Total carbohydrate in the soil extract was determined by phenol-method without acid hydrolysis (Safarik and Santruckova, 1992). The extracted soil solution was mixed with $5 \%$ phenol solution on a 1:1 ratio, and the absorbance measured at $485 \mathrm{~nm}$ with glucose as standard. Results are expressed on an oven dry basis. The activity of two soil enzymes, $\beta$-glucosidase and acid phosphatase, was determined using the methods described by Tabatabai (1994). Field moist sieved samples stored at $4{ }^{\circ} \mathrm{C}$ for 12 weeks after sampling were used for the enzyme assays. Results are expressed on the basis of oven dry soil $\left(105^{\circ} \mathrm{C}\right)$. Water content was determined after oven-drying at $105{ }^{\circ} \mathrm{C}$ for $48 \mathrm{~h}$. Statistical analysis was based on simple linear regression and analysis of variance. The data in all tables are given as the mean \pm standard error of the six treatments, unless indicated otherwise.

\section{RESULTS AND DISCUSSION}

\subsection{Soil pH and bulk density}

The effect of cultivation and straw disposal treatment on soil $\mathrm{pH}$ and bulk density is shown in Table II. Topsoil pH varied with tillage treatment, but this effect was not observed in the lower horizons. Soil $\mathrm{pH}$ at $50 \mathrm{~mm}$ depth under mouldboard ploughing was greater than the other two tillage treatments, and increased significantly with soil depth, but there was no significant effect of straw management on $\mathrm{pH}$ (Tab. III). These results are in agreement with most previous studies which have mostly reported non-significant effects of cultivation systems
Table II. Effect of tillage method and straw disposal treatment on soil $\mathrm{pH}$ and bulk density $(\mathrm{n}=4)$.

\begin{tabular}{lcccccc}
\hline & \multicolumn{5}{c}{ Soil pH } & \multicolumn{5}{c}{ Bulk density $\left(\mathrm{Mg} \mathrm{m}^{-3}\right)$} \\
\cline { 2 - 7 } & 50 & 150 & 250 & 50 & 150 & 250 \\
\cline { 2 - 7 } & 6.82 & 7.77 & 8.13 & 1.08 & 1.19 & 1.23 \\
\hline $\begin{array}{l}\text { Chisel plough, straw } \\
\text { incorporated }\end{array}$ & 7.34 & 8.06 & 8.26 & 1.15 & 1.29 & 1.32 \\
$\begin{array}{l}\text { Chisel plough, straw } \\
\text { removed }\end{array}$ & 7.51 & 7.67 & 7.85 & 1.09 & 1.16 & 1.23 \\
$\begin{array}{l}\text { Mouldboard, straw } \\
\text { incorporated }\end{array}$ & 7.70 & 7.85 & 8.03 & 1.11 & 1.19 & 1.32 \\
$\begin{array}{l}\text { Mouldboard, straw } \\
\text { removed }\end{array}$ & 6.67 & 8.14 & 8.21 & 1.20 & 1.22 & 1.32 \\
$\begin{array}{l}\text { No-till, straw } \\
\begin{array}{l}\text { incorporated } \\
\text { No-till, straw removed }\end{array}\end{array}$ & 6.80 & 7.95 & 8.15 & 1.21 & 1.26 & 1.32 \\
\hline
\end{tabular}

on pH (Dalal et al., 1991) Straw incorporation caused a 2.3$5.0 \%$ bulk density reduction at the 150 and $250 \mathrm{~mm}$ depths, while similar effect was not observed at $50 \mathrm{~mm}$. In comparison to mouldboard ploughing, chisel ploughing and no-till caused an $8-11 \%$ and $5 \%$ bulk density increase at 50 and $150 \mathrm{~mm}$, respectively. However, in case of the $250 \mathrm{~mm}$ depth, no-till and chisel ploughing had a beneficial effect on bulk density, causing a 3.4-6.6\% decrease compared to mouldboard ploughing (Tab. II). These observations agree with many previous studies which note that despite frequent loosening by cultivation, the declining soil structural condition of mouldboard ploughed soils usually leads to increased compaction, particularly after many years of contrasting cultivation (e.g. Carter, 2005; He et al., 2009).

\subsection{Soil organic carbon, nitrogen and $\mathrm{C} / \mathrm{N}$ ratio}

Soil organic carbon concentration varied with tillage and straw disposal treatment (Fig. 1b). SOC was significantly higher with chisel ploughing and no-till cultivation compared with mouldboard ploughing at the $50 \mathrm{~mm}$ soil depth, while a reverse trend was observed at 150 and $250 \mathrm{~mm}$ (Tabs. III, IV). Chisel ploughing and no-till cultivation, together with straw incorporation significantly increased the SOC at $50 \mathrm{~mm}$ compared with mouldboard plough without straw incorporation. The largest increase occurred in no-till straw incorporated treatment $(17.7 \%)$ and the smallest was recorded in the no-till straw removed treatment (7.1\%). In general, straw incorporation did not have any effect on concentration of SOC in the lower soil horizons (150 and $250 \mathrm{~mm}$ ). The SOC concentration at depths of 150 and $250 \mathrm{~mm}$ were consistently lower than at $50 \mathrm{~mm}$. Irrespective of soil depth, there was no significant interaction effect of tillage and straw treatments on SOC, while soil depth did have a significant effect on SOC (Tab. IV). In order to quantify the mass of SOC for each treatment it is necessary to account for variation in bulk density (Ellert and Bettany, 1995). Following adjustment using mean bulk density values for each treatment to calculate total C stock, tillage and straw management was seen to have a significant effect 
Table III. Statistical summary of tillage (T) and straw management (S) effect on soil properties. F-values and significance level among means of different soil properties were computed by two way ANOVA with factors tillage $(d f=2)$ and straw management $(d f=1)$. Values within brackets represent least significant difference $(P<0.05)$. * $P<0.05$, ** $P<0.01$, *** $P<0.001$.

\begin{tabular}{|c|c|c|c|c|c|c|c|c|c|}
\hline \multirow{2}{*}{ Depth $(\mathrm{mm})$} & \multicolumn{3}{|c|}{50} & \multicolumn{3}{|c|}{150} & \multicolumn{3}{|c|}{250} \\
\hline & Tillage & Straw & $\begin{array}{c}\mathrm{T} \times \mathrm{S} \\
\text { interaction }\end{array}$ & Tillage & Straw & $\begin{array}{c}\mathrm{T} \times \mathrm{S} \\
\text { interaction }\end{array}$ & Tillage & Straw & $\begin{array}{c}\mathrm{T} \times \mathrm{S} \\
\text { interaction }\end{array}$ \\
\hline $\begin{array}{l}\text { Total nitrogen } \\
(\%)\end{array}$ & $\begin{array}{c}1.58 \\
(0.02)\end{array}$ & $\begin{array}{c}2.06 \\
(0.017)\end{array}$ & $\begin{array}{c}1.57 \\
(0.029)\end{array}$ & $\begin{array}{c}2.15 \\
(0.017)\end{array}$ & $\begin{array}{c}0.84 \\
(0.014)\end{array}$ & $\begin{array}{c}1.37 \\
(0.023)\end{array}$ & $\begin{array}{c}15.94 * * * \\
(0.023)\end{array}$ & $\begin{array}{c}6.38 * \\
(0.018)\end{array}$ & $\begin{array}{c}1.27 \\
(0.032)\end{array}$ \\
\hline $\begin{array}{l}\text { Soil organic carbon } \\
(\%)\end{array}$ & $\begin{array}{l}5.22 * \\
(0.21)\end{array}$ & $\begin{array}{l}7.87 * \\
(0.17)\end{array}$ & $\begin{array}{c}1.68 \\
(0.29)\end{array}$ & $\begin{array}{c}3.20 \\
(0.25)\end{array}$ & $\begin{array}{c}0.09 \\
(0.21)\end{array}$ & $\begin{array}{c}0.85 \\
(0.36)\end{array}$ & $\begin{array}{c}12.48 * * \\
(0.31)\end{array}$ & $\begin{array}{c}2.54 \\
(0.25)\end{array}$ & $\begin{array}{c}0.56 \\
(0.44)\end{array}$ \\
\hline $\mathrm{C} / \mathrm{N}$ ratio & $\begin{array}{c}12.61 * * \\
(0.18)\end{array}$ & $\begin{array}{l}21.19 * * * \\
(0.15)\end{array}$ & $\begin{array}{c}0.69 \\
(0.26)\end{array}$ & $\begin{array}{l}4.13 * \\
(0.36)\end{array}$ & $\begin{array}{l}0.17 \\
(0.29)\end{array}$ & $\begin{array}{c}0.68 \\
(0.51)\end{array}$ & $\begin{array}{l}6.27 * \\
(0.46)\end{array}$ & $\begin{array}{c}0.14 \\
(0.37)\end{array}$ & $\begin{array}{c}0.10 \\
(0.65)\end{array}$ \\
\hline $\begin{array}{l}\text { Bulk density } \\
\left(\mathrm{Mg} \mathrm{m}^{-3}\right)\end{array}$ & $\begin{array}{l}6.26^{*} \\
(0.72)\end{array}$ & $\begin{array}{c}1.30 \\
(0.06)\end{array}$ & $\begin{array}{c}0.66 \\
(0.10)\end{array}$ & $\begin{array}{l}3.65 \\
(0.06)\end{array}$ & $\begin{array}{l}7.58^{*} \\
(0.05)\end{array}$ & $\begin{array}{c}0.93 \\
(0.08)\end{array}$ & $\begin{array}{l}6.26^{*} \\
(0.055)\end{array}$ & $\begin{array}{c}2.09 \\
(0.045)\end{array}$ & $\begin{array}{l}8.64 * * \\
(0.078)\end{array}$ \\
\hline $\begin{array}{l}\text { Nitrogen stock } \\
\left(\mathrm{Mg} \mathrm{ha}^{-1}\right)\end{array}$ & $\begin{array}{c}1.74 \\
(0.252)\end{array}$ & $\begin{array}{c}2.03 \\
(0.205)\end{array}$ & $\begin{array}{c}1.95 \\
(0.356)\end{array}$ & $\begin{array}{c}1.92 \\
(0.23)\end{array}$ & $\begin{array}{c}1.21 \\
(0.188)\end{array}$ & $\begin{array}{c}1.27 \\
(0.325)\end{array}$ & - & - & - \\
\hline $\begin{array}{l}\text { Carbon stock } \\
\left(\mathrm{Mg} \mathrm{ha}^{-1}\right)\end{array}$ & $\begin{array}{l}5.30 * \\
(2.50)\end{array}$ & $\begin{array}{l}6.62 * \\
(2.04)\end{array}$ & $\begin{array}{c}1.04 \\
(3.54)\end{array}$ & $\begin{array}{c}0.82 \\
(4.55)\end{array}$ & $\begin{array}{c}0.23 \\
(3.71)\end{array}$ & $\begin{array}{c}0.96 \\
(6.44)\end{array}$ & - & - & - \\
\hline $\mathrm{pH}$ & $\begin{array}{l}8.43 * * \\
(0.47)\end{array}$ & $\begin{array}{c}2.66 \\
(0.39)\end{array}$ & $\begin{array}{c}0.50 \\
(0.67)\end{array}$ & $\begin{array}{c}1.48 \\
(0.37)\end{array}$ & $\begin{array}{c}0.47 \\
(0.30)\end{array}$ & $\begin{array}{c}1.14 \\
(0.52)\end{array}$ & $\begin{array}{c}2.39 \\
(0.29)\end{array}$ & $\begin{array}{c}0.62 \\
(0.24)\end{array}$ & $\begin{array}{c}0.51 \\
(0.41)\end{array}$ \\
\hline $\begin{array}{l}\text { Water extractable organic carbon } \\
(\%)\end{array}$ & $\begin{array}{c}2.08 \\
(0.0008)\end{array}$ & $\begin{array}{c}5.91 * \\
(0.0006)\end{array}$ & $\begin{array}{c}0.30 \\
(0.0011)\end{array}$ & $\begin{array}{c}1.0 \\
(0.0008)\end{array}$ & $\begin{array}{c}0.15 \\
(0.0007)\end{array}$ & $\begin{array}{c}0.56 \\
(0.0012)\end{array}$ & $\begin{array}{c}0.02 \\
(0.0018)\end{array}$ & $\begin{array}{c}0.34 \\
(0.0015)\end{array}$ & $\begin{array}{c}0.10 \\
(0.0025)\end{array}$ \\
\hline $\begin{array}{l}\text { Hot water soluble carbon } \\
\left(\mu \mathrm{g} \mathrm{C} \mathrm{g}^{-1} \text { soil }\right)\end{array}$ & $\begin{array}{l}57.23 * * * \\
(25.74)\end{array}$ & $\begin{array}{l}46.42 * * * \\
(21.10)\end{array}$ & $\begin{array}{c}0.86 \\
(36.41)\end{array}$ & $\begin{array}{c}27.74 * * * \\
(31.89)\end{array}$ & $\begin{array}{c}3.92 \\
(26.04)\end{array}$ & $\begin{array}{c}1.87 \\
(45.10)\end{array}$ & $\begin{array}{c}63.86 * * * \\
(22.30)\end{array}$ & $\begin{array}{c}7.43^{*} \\
(18.21)\end{array}$ & $\begin{array}{c}1.23 \\
(31.54)\end{array}$ \\
\hline $\begin{array}{l}\text { Microbial biomass carbon } \\
\left(\mu \mathrm{g} \mathrm{Cg}^{-1} \text { soil }\right)\end{array}$ & $\begin{array}{c}2.81 \\
(111.1)\end{array}$ & $\begin{array}{c}3.85 \\
(90.70)\end{array}$ & $\begin{array}{c}0.13 \\
(157.1)\end{array}$ & $\begin{array}{c}0.79 \\
(156.45)\end{array}$ & $\begin{array}{c}1.72 \\
127.74)\end{array}$ & $\begin{array}{c}0.27 \\
(221.25)\end{array}$ & $\begin{array}{c}9.71 * * \\
(159.30)\end{array}$ & $\begin{array}{c}0.21 \\
(130.07)\end{array}$ & $\begin{array}{c}1.15 \\
(225.28)\end{array}$ \\
\hline $\begin{array}{l}\text { Microbial biomass carbon/ } \\
\text { Soil organic carbon }\end{array}$ & $\begin{array}{c}1.04 \\
(0.0022)\end{array}$ & $\begin{array}{c}0.58 \\
(0.0018)\end{array}$ & $\begin{array}{c}0.74 \\
(0.0031)\end{array}$ & $\begin{array}{c}0.78 \\
(0.0043)\end{array}$ & $\begin{array}{c}1.89 \\
(0.0035)\end{array}$ & $\begin{array}{c}0.92 \\
(0.0061)\end{array}$ & $\begin{array}{c}0.98 \\
(0.0073)\end{array}$ & $\begin{array}{c}1.55 \\
(0.006)\end{array}$ & $\begin{array}{c}1.86 \\
(0.0103)\end{array}$ \\
\hline $\begin{array}{l}\text { Water extractable carbon/ } \\
\text { Soil organic carbon }\end{array}$ & $\begin{array}{l}1.68 \\
(0.022)\end{array}$ & $\begin{array}{l}5.00^{*} \\
(0.018)\end{array}$ & $\begin{array}{c}0.19 \\
(0.031)\end{array}$ & $\begin{array}{c}1.86 \\
(0.024)\end{array}$ & $\begin{array}{c}0.15 \\
((0.02)\end{array}$ & $\begin{array}{c}0.38 \\
(0.034)\end{array}$ & $\begin{array}{c}0.29 \\
(0.065)\end{array}$ & $\begin{array}{c}0.19 \\
(0.053)\end{array}$ & $\begin{array}{c}0.011 \\
(0.092)\end{array}$ \\
\hline $\begin{array}{l}\beta \text {-glucosidase } \\
\left(\mu \mathrm{g} \text { p-nitrophenol } \mathrm{g}^{-1} \text { soil }\right)\end{array}$ & $\begin{array}{l}11.32 * * \\
(29.65)\end{array}$ & $\begin{array}{l}13.26 * * \\
(24.20)\end{array}$ & $\begin{array}{c}0.30 \\
(41.93)\end{array}$ & $\begin{array}{l}15.74 * * * \\
(29.49)\end{array}$ & $\begin{array}{l}8.81 * * \\
(24.08)\end{array}$ & $\begin{array}{c}2.24 \\
(41.70)\end{array}$ & $\begin{array}{l}28.41 * * * \\
(21.45)\end{array}$ & $\begin{array}{c}5.14 * \\
(17.51)\end{array}$ & $\begin{array}{c}1.77 \\
(30.34)\end{array}$ \\
\hline $\begin{array}{l}\text { Acid phosphatase } \\
\left(\mu \mathrm{g} \text { p-nitrophenol } \mathrm{g}^{-1} \text { soil }\right)\end{array}$ & $\begin{array}{l}14.46 * * \\
(34.99)\end{array}$ & $\begin{array}{l}14.36 * * \\
(28.57)\end{array}$ & $\begin{array}{c}5.62 * \\
(49.49)\end{array}$ & $\begin{array}{c}1.06 \\
(22.84)\end{array}$ & $\begin{array}{c}4.46 \\
(18.65)\end{array}$ & $\begin{array}{c}1.93 \\
(32.30)\end{array}$ & $\begin{array}{l}11.13 * * \\
(38.51)\end{array}$ & $\begin{array}{c}0.28 \\
(31.44)\end{array}$ & $\begin{array}{c}0.27 \\
(54.46)\end{array}$ \\
\hline$\delta^{12} \mathrm{C}$ & $\begin{array}{c}38.32 * * * \\
(0.388)\end{array}$ & $\begin{array}{c}27.36^{* * * *} \\
(0.317)\end{array}$ & $\begin{array}{c}11.63 * * \\
(0.548)\end{array}$ & $\begin{array}{c}3.04 \\
(0.413)\end{array}$ & $\begin{array}{c}0.94 \\
(0.337)\end{array}$ & $\begin{array}{c}0.14 \\
(0.584)\end{array}$ & $\begin{array}{c}7.23 * \\
(0.395)\end{array}$ & $\begin{array}{c}5.70^{*} \\
(0.322)\end{array}$ & $\begin{array}{c}0.46 \\
(0.558)\end{array}$ \\
\hline$\delta^{15} \mathrm{~N}$ & $\begin{array}{c}10.42 * * \\
(0.53)\end{array}$ & $\begin{array}{c}4.20 \\
(0.43)\end{array}$ & $\begin{array}{l}7.29 * \\
(0.75)\end{array}$ & $\begin{array}{c}30.97 * * * \\
(0.143)\end{array}$ & $\begin{array}{c}1.10 \\
(0.117)\end{array}$ & $\begin{array}{c}1.28 \\
(0.202)\end{array}$ & $\begin{array}{c}72.07 * * * \\
(0.161)\end{array}$ & $\begin{array}{c}0.120 \\
(0.131)\end{array}$ & $\begin{array}{c}29.70 * * * \\
(0.227)\end{array}$ \\
\hline
\end{tabular}

on $\mathrm{C}$ stock at $50 \mathrm{~mm}$. After twelve years, the carbon stock of the soil at the $50 \mathrm{~mm}$ depth under no-till straw incorporation and chisel ploughing with straw incorporation increased by 17 and $14 \%$ respectively, compared with the soil under mouldboard ploughing with straw removed (Tab. V).

While there was no observed effect of tillage method or straw incorporation on total $\mathrm{N}$ at 50 or $150 \mathrm{~mm}$ soil depths (Fig. 1a, Tab. III), at $250 \mathrm{~mm}$ total $\mathrm{N}$ under mouldboard ploughing was significantly higher than the other cultivation methods. Straw incorporation also resulted in a significant increase in total $\mathrm{N}$. The effect of straw management on $\mathrm{C} / \mathrm{N}$ ratio was evident only in the topsoil, where straw incorporation resulted in an increase in the $\mathrm{C} / \mathrm{N}$ ratio. Tillage had a significant effect on the $\mathrm{C} / \mathrm{N}$ ratio in the topsoil with the highest value
(8.75) observed in the no-till soil followed by chisel (8.65) and mouldboard ploughing (8.35). At the 150 and $250 \mathrm{~mm}$ depths, the opposite trend was observed, with the highest value under mouldboard ploughing followed by chisel ploughing and notill. The summary statistical significance of these differences is given in Table IV.

The results reported here confirm the importance of using data from long term tillage experiments to assess changes in soil organic matter and related properties. The increase in carbon stock following no-till and chisel ploughing that was confined to the $50 \mathrm{~mm}$ soil depth, and was not apparent for the combined sampling depth of 0-200 $\mathrm{mm}$. The widespread view that non-inversion tillage favours carbon sequestration may simply be an artefact of sampling methodology. For example, 
(a)

Total N (\%)

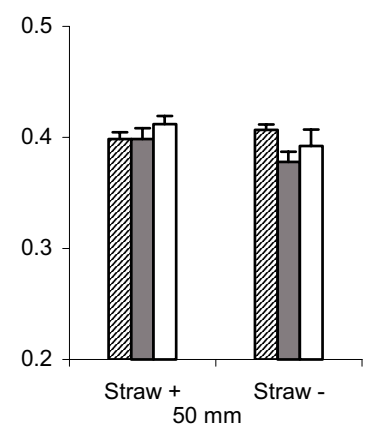

(b)

Soil organic carbon (\%)

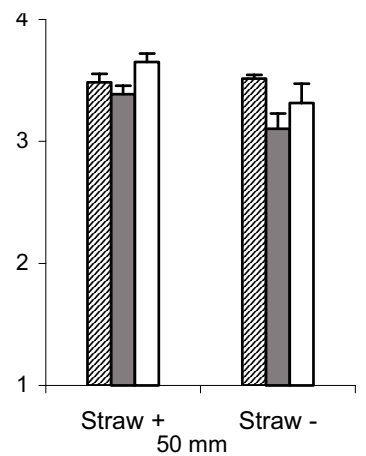

(c)

Microbial biomass carbon ( $\mu \mathrm{C} \mathrm{g} \mathrm{g}^{-1}$ soil)

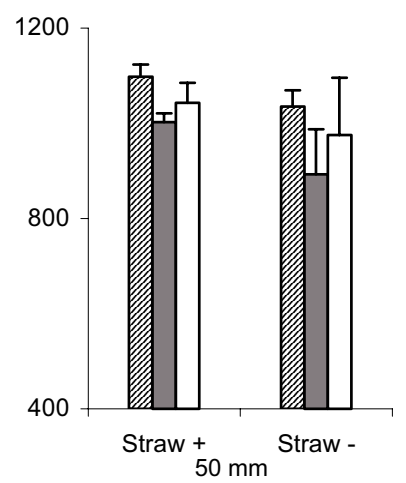

国 Chisel $\square$ Mouldboard $\square$ No-till
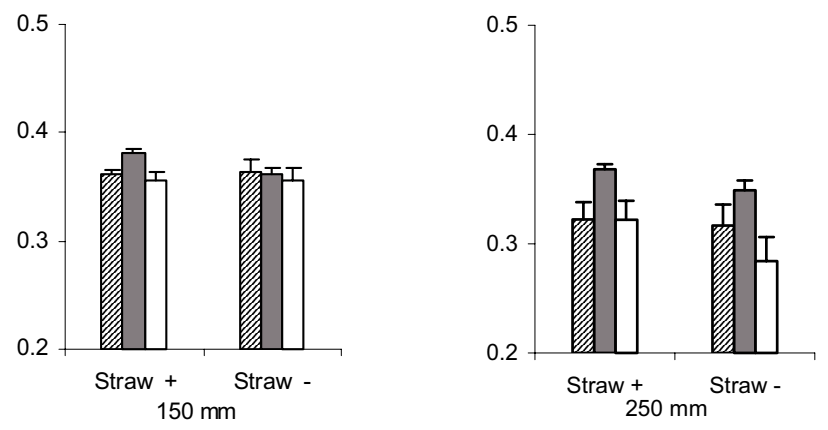
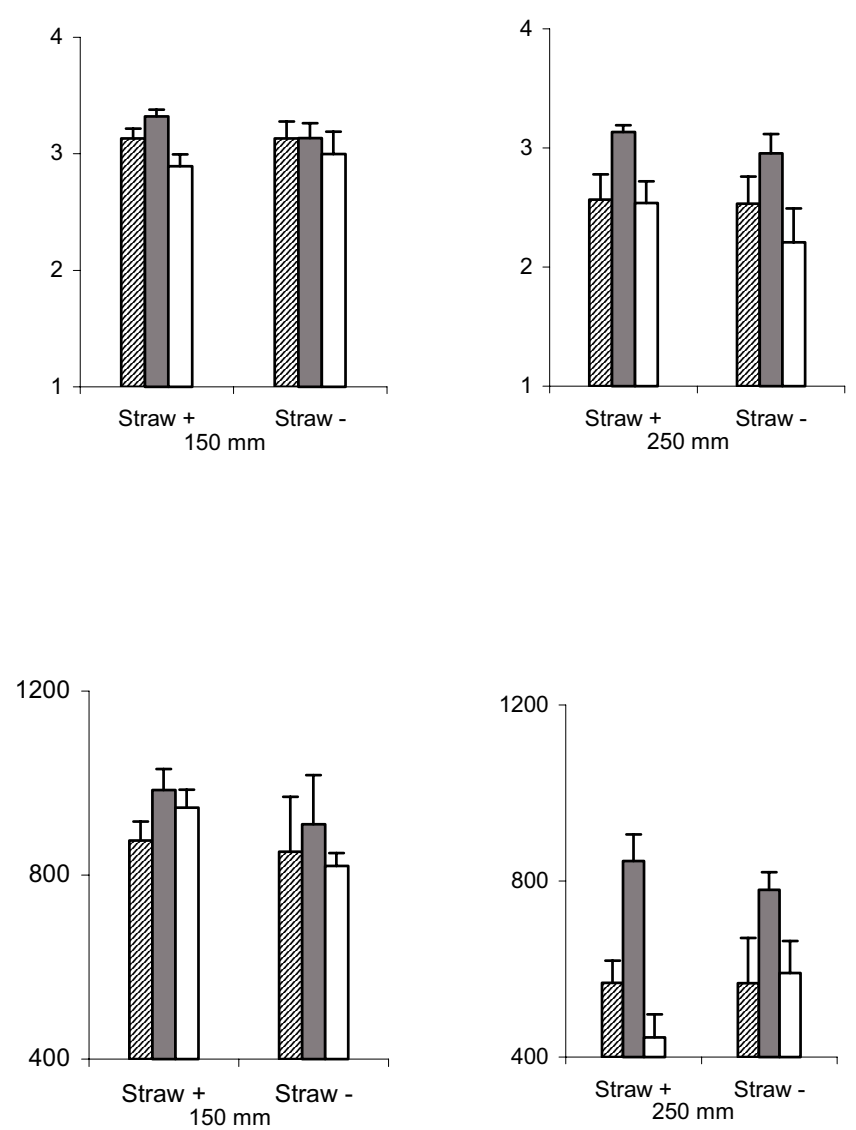

Figure 1. Effect of cultivation method (chisel plough, mouldboard plough and no-till) and straw management $(+=$ incorporated, $-=$ removed) on the concentration of (a) total nitrogen, (b) soil organic carbon and (c) microbial biomass carbon at 50, 150 and $250 \mathrm{~mm}$ soil depth. Highest total nitrogen, soil organic carbon and microbial biomass carbon were observed in the $50 \mathrm{~mm}$ soil depth under all treatments. Deep mixing of the mouldboard ploughed plots resulted in higher concentrations of total nitrogen, soil organic carbon and particularly microbial biomass carbon at the $250 \mathrm{~mm}$ depth. The effect of straw incorporation (Straw+) was most noticeable on the no-till plots, demonstrating the positive impact that conservation tillage can have on soil surface conditions. 
Table IV. Statistical summary of tillage and depth effect on soil properties (F-value and significance level) computed by two way ANOVA. Values within brackets represent the least significant difference $(P<0.05)$. $* P<0.05, * * P<0.01, * * * P<0.001$.

\begin{tabular}{|c|c|c|c|}
\hline & $\begin{array}{l}\text { Tillage } \\
(d f=2)\end{array}$ & $\begin{array}{c}\text { Depth } \\
(d f=2)^{\#}\end{array}$ & $\begin{array}{c}\text { Tillage } \times \text { Depth } \\
\text { interaction } \\
(d f=10)\end{array}$ \\
\hline Total nitrogen & $6.47 * *$ & $88.77 * * *$ & $7.90 * * *$ \\
\hline$(\%)$ & $(0.01)$ & $(0.01)$ & $(0.019)$ \\
\hline Soil organic carbon & $6.83 * *$ & $69.51 * * *$ & $9.59 * * *$ \\
\hline$(\%)$ & $(0.13)$ & $(0.13)$ & $(0.23)$ \\
\hline $\mathrm{C} / \mathrm{N}$ ratio & $\begin{array}{l}5.26 * * \\
(0.18)\end{array}$ & $\begin{array}{c}20.66^{* * * *} \\
(0.18)\end{array}$ & $\begin{array}{c}7.14 * * * \\
(0.31)\end{array}$ \\
\hline Bulk density & $14.29 * *$ & $37.20 * * *$ & 1.31 \\
\hline$\left(\mathrm{Mg} \mathrm{m}^{-3}\right)$ & $(0.03)$ & $(0.03)$ & $(0.06)$ \\
\hline Nitrogen stock & 0.14 & $4.37 *$ & 1.17 \\
\hline$\left(\mathrm{Mg} \mathrm{ha}^{-1}\right)$ & $(0.20)$ & $(0.17)$ & $(0.29)$ \\
\hline Carbon stock & 0.81 & 3.61 & $4.00 *$ \\
\hline$\left(\mathrm{Mg} \mathrm{ha}^{-1}\right)$ & $(2.20)$ & $(1.80)$ & $(3.12)$ \\
\hline $\mathrm{pH}(1: 2.5)$ & $\begin{array}{c}1.02 \\
(0.16)\end{array}$ & $\begin{array}{c}80.20 * * * \\
(0.16)\end{array}$ & $\begin{array}{l}11.37 * \\
(0.28)\end{array}$ \\
\hline $\begin{array}{l}\text { Water extractable carbon } \\
(\%)\end{array}$ & $\begin{array}{c}0.35 \\
(0.0006)\end{array}$ & $\begin{array}{c}13.49 * * * \\
(0.0006)\end{array}$ & $\begin{array}{c}0.63 \\
(0.001)\end{array}$ \\
\hline $\begin{array}{l}\text { Hot water extractable carbohydrate } \\
\left(\mu \mathrm{g} \mathrm{C}^{-1} \text { soil) }\right.\end{array}$ & $\begin{array}{c}8.73 * * * \\
(15.41)\end{array}$ & $\begin{array}{l}530 * * * \\
(15.41)\end{array}$ & $\begin{array}{l}52.19 * * * \\
(26.69)\end{array}$ \\
\hline $\begin{array}{l}\text { Microbial biomass carbon } \\
\left(\mu \mathrm{g} \mathrm{C}^{-1} \text { soil) }\right.\end{array}$ & $\begin{array}{l}3.41 * \\
(78.95)\end{array}$ & $\begin{array}{c}48.66 * * * \\
(78.95)\end{array}$ & $\begin{array}{c}4.91 * * \\
(136.75)\end{array}$ \\
\hline Microbial biomass carbon/ & 0.54 & $8.13^{* *}$ & 0.83 \\
\hline Soil organic carbon & $(0.003)$ & $(0.003)$ & $(0.005)$ \\
\hline Water extractable carbon/ & 1.51 & $24.79 * * *$ & 0.36 \\
\hline Soil organic carbon & $(0.02)$ & $(0.02)$ & $(0.034)$ \\
\hline$\beta$-glucosidase & $8.05 * *$ & $196.56^{* * *}$ & $20.76 * * *$ \\
\hline ( $\mu \mathrm{g} p$-nitrophenol $\mathrm{g}^{-1}$ soil) & (14.25) & $(14.25)$ & $(24.69)$ \\
\hline $\begin{array}{l}\text { Acid phosphatase } \\
\text { ( } \mu \mathrm{g} p \text {-nitrophenol } \mathrm{g}^{-1} \text { soil) }\end{array}$ & $\begin{array}{c}0.28 \\
(19.85)\end{array}$ & $\begin{array}{l}83.99 * * * \\
(19.85)\end{array}$ & $\begin{array}{l}11.96 * * * \\
(34.37)\end{array}$ \\
\hline$\delta^{13} \mathrm{C}$ & $\begin{array}{c}13.64 * * * \\
(0.24)\end{array}$ & $\begin{array}{c}22.28 * * * \\
(0.24)\end{array}$ & $\begin{array}{c}10.77 * * * \\
(0.42)\end{array}$ \\
\hline$\delta^{15} \mathrm{~N}$ & $\begin{array}{c}1.53 \\
(0.26)\end{array}$ & $\begin{array}{c}6.55 * * \\
(0.26)\end{array}$ & $\begin{array}{c}10.57 * * * \\
(0.44)\end{array}$ \\
\hline
\end{tabular}

$\# d f=1$ for $\mathrm{C}, \mathrm{N}$ and microbial biomass carbon stock.

He et al. (2009) observed statistically significant differences between cultivation systems at soil depths down to $200 \mathrm{~mm}$, but not deeper. Studies that have involved deeper sampling generally show no $\mathrm{C}$ sequestration advantage for minimum tillage, and often show more $\mathrm{C}$ in conventionally tilled systems. In a review of $\mathrm{C}$ sequestration research in Canada, Vanden Bygaart et al. (2003) reported nearly 100 plot studies of the impact of conservation tillage in Canada. In 17 experiments where the sampling depth was $300 \mathrm{~mm}$ or less, 37 out of 45 no-till treatments $(82 \%)$ reported more SOC than in the conventionally tilled (mouldboard ploughed) control, with a mean annual SOC gain of $0.38 \pm 0.72 \mathrm{t} \mathrm{ha}^{-1}$ year $^{-1}$; in the five experiments where the profile was sampled to a depth greater than $300 \mathrm{~mm}$, a majority of the trials (35 out of 51, or $69 \%$ ) registered less SOC in the no-till treatment relative to conventional tillage, with a mean annual SOC loss of $-0.23 \pm$ $0.97 \mathrm{tha}^{-1}$ year $^{-1}$. In this review even a shallow combined sampling depth of 0-200 $\mathrm{mm}$ did not show any advantage of having more sequestration of carbon under non-inversion tillage compared with inversion tillage. Vanden Bygaart et al. (2003) observe that non-inversion tillage physically protects part of the organic matter in the top layer from mineralization by inclusion within macro-aggregates. With conventional inversion tillage on the other hand, aggregates will be more thoroughly disrupted, assisting loss of organic matter (Rasmussen and Collins, 1991). In our experiment chisel ploughing and notill increased the $\mathrm{C} / \mathrm{N}$ ratio at the $50 \mathrm{~mm}$ depth due to the straw becoming concentrated on the soil surface while mouldboard ploughing increased the $\mathrm{C} / \mathrm{N}$ ratio at $250 \mathrm{~mm}$ due to buried particulate organic material.

\subsection{Labile soil organic matter and soil enzyme activity}

Long-term tillage treatments had a significant effect on the distribution of microbial biomass carbon with the highest values observed under mouldboard ploughing (Fig. 1c and 
Table V. Effect of tillage and straw disposal treatment on selected soil quality characteristics and soil organic carbon stock (note: data not available for SOC at $250 \mathrm{~mm}$ depth, see discussion in text).

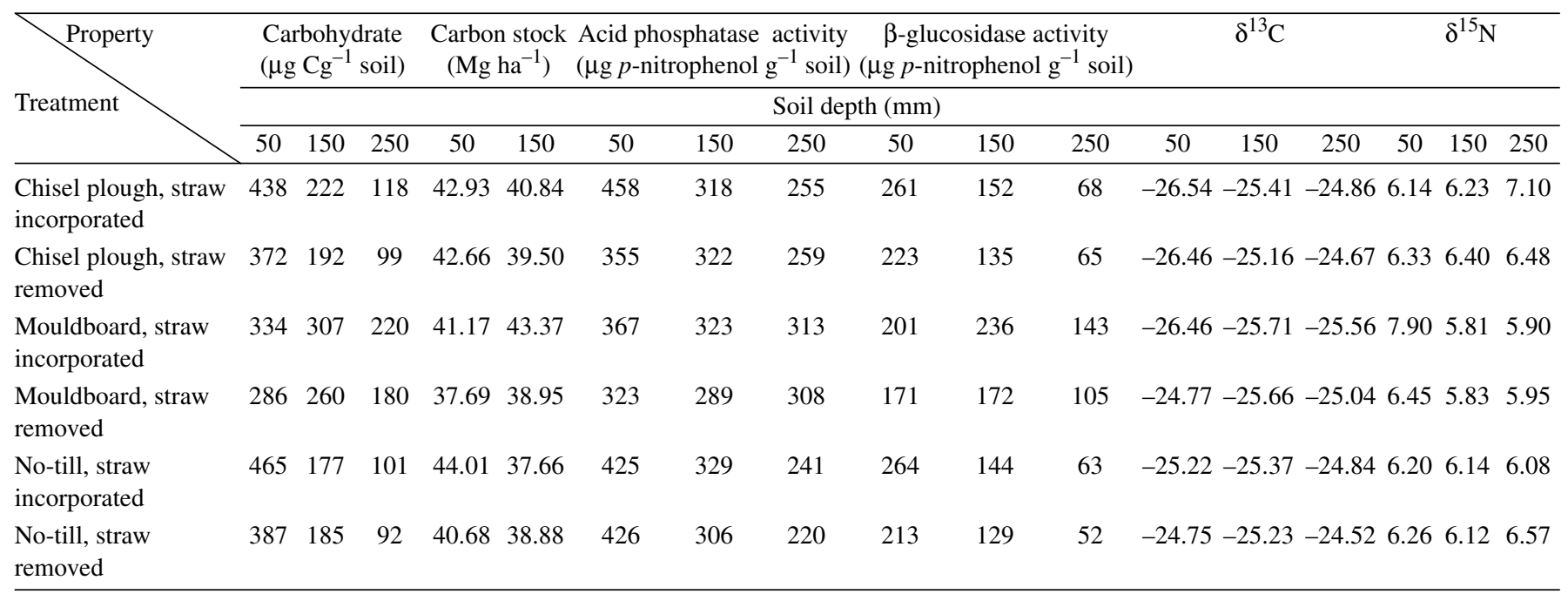

Tabs. III, IV). At $50 \mathrm{~mm}$, adoption of no-till and chisel ploughing did not produce a significant change in the concentration of microbial biomass carbon. Straw incorporation also did not have any significant effect on microbial biomass carbon, although the distribution of microbial biomass carbon within the sampling depth was significantly affected by treatment. A gradient of microbial biomass carbon was evident down the soil profile, but the total was not statistically different between tillage systems.

Straw incorporation significantly increased the water extractable carbon at $50 \mathrm{~mm}$, but this effect was not observed at 150 or $250 \mathrm{~mm}$ (Tab. III), nor was there any interaction effect of tillage and straw management on water extractable organic carbon. Tillage system strongly influenced the hot water extractable carbohydrate concentration of soil at all sampling depths (Tab. III). As with SOC, hot water extractable carbohydrate was concentrated in the $50 \mathrm{~mm}$ layer under no-till and chisel ploughing, but was more uniformly distributed down profile under mouldboard ploughing. The concentration of hot water extractable carbohydrate at $50 \mathrm{~mm}$ increased under all the tillage treatments after twelve years of straw incorporation, being highest for the no-till soil (426 $\left.\mu \mathrm{g} \mathrm{C} \mathrm{g}^{-1}\right)$ compared with chisel (405 $\mu \mathrm{g} \mathrm{C} \mathrm{g}^{-1}$ ) and mouldboard ploughing $\left(310 \mu \mathrm{g} \mathrm{C} \mathrm{g}^{-1}\right)$.

The activities of $\beta$-glucosidase and acid phosphatase enzymes in different depths of soil under different tillage and straw management are shown in Table V. In general, chisel ploughing and no-till increased enzyme activity at $50 \mathrm{~mm}$, where as mouldboard ploughing tended to increase enzyme activity in the subsurface layers. With one exception, the tillage treatments had a significant effect on the activities of both enzymes in all the depths of soil. Straw incorporation had a marked effect on $\beta$-glucosidase activity in all the depths of soil whereas the effect was confined only to the $50 \mathrm{~mm}$ soil depth for acid phosphatase (Tab. V). No-till and chisel plough- ing in association with straw incorporation increased enzyme activity at $50 \mathrm{~mm}$ compared with mouldboard ploughing. The activities of the enzymes declined with soil depth, and there was strong interaction effect between tillage and soil depth on the two soil enzymes studied. The regression relationships between enzyme activities, other soil properties, tillage and straw treatments for the whole soil profile are given in Table VI.

The reduction of tillage intensity from mouldboard ploughing to either chisel ploughing or no-till, as well as straw incorporation increased the supply of carbohydrates for microorganisms and soil enzyme activity. Enhanced microbial activity was reflected by increased microbial biomass carbon concentration, and its significant positive correlation (Tab. VI) with $\beta$-glucosidase activity in soil. The highly significant effect of tillage and straw management on $\beta$-glucosidase activity, along with the high correlation between this enzyme and microbial biomass carbon, suggests that $\beta$-glucosidase enzyme is a good indicator to assess the effect of long term tillage and straw management on the biological activity of soil.

The effects of tillage reduction and straw incorporation were only seen in differing soil $\delta^{13} \mathrm{C}$, but not in $\delta^{15} \mathrm{~N}$ ratio under the various treatments. The absence of required long term crop and incorporated straw $\delta^{13} \mathrm{C}$ and $\delta^{15} \mathrm{~N}$ data makes it difficult to explain the soil isotope results further, as was achieved in some previous studies (e.g. Wanniarachchi et al., 1999). However, the results confirm that $\delta^{13} \mathrm{C}$ is indeed a good diagnostic indicator of long term management induced changes on organic matter content and distribution in arable soils, in line with findings in other studies (e.g. Lobe et al., 2005). Microbial biomass carbon and its ratio with SOC have been suggested as useful measures for assessing organic matter dynamics (Sparling, 1992). The variation of microbial biomass carbon with depth under non-inversion and inversion tillage reported here was consistent with previous research (e.g. Deng and Tabatabai, 1997). Water extractable carbon, being a highly 
Table VI. Relationship between selected soil properties, tillage and straw management for the 0-300 mm soil profile.

\begin{tabular}{lccc}
\hline & Variables & Equation & $\mathrm{r}$ \\
\hline Dependent & Independent & & \\
\hline Hot water soluble carbohydrate & Soil organic carbon & $\mathrm{y}=0.22 \mathrm{x}-0.43$ & $0.83^{* * *}$ \\
Total N & Soil organic carbon & $\mathrm{y}=0.085 \mathrm{x}+0.104$ & $0.98^{* * *}$ \\
Microbial biomass carbon & Soil organic carbon & $\mathrm{y}=0.035 \mathrm{x}-0.024$ & $0.74 * * *$ \\
Microbial biomass carbon & Hot water soluble carbohydrate & $\mathrm{y}=1.4 \mathrm{x}+501.7$ & $0.78^{* * *}$ \\
Bulk density & Soil organic carbon & $\mathrm{y}=-0.12 \mathrm{x}+1.57$ & $0.63^{* * *}$ \\
Bulk density & Hot water soluble carbohydrate & $\mathrm{y}=-0.0005 \mathrm{x}+1.33$ & $0.68^{* * *}$ \\
Acid phosphatase & Microbial biomass carbon & $\mathrm{y}=2287.5 \mathrm{x}+131$ & $0.71^{* * *}$ \\
Acid phosphatase & Hot water soluble carbohydrate & $\mathrm{y}=0.51 \mathrm{x}+199.5$ & $0.88^{* * *}$ \\
Acid phosphatase & pH & $\mathrm{y}=-82.1 \mathrm{x}+957.8$ & $0.71^{* * *}$ \\
Acid phosphatase & Soil organic carbon & $\mathrm{y}=128.2 \mathrm{x}-67.5$ & $0.83^{* * *}$ \\
$\beta-$ glucosidase & Microbial biomass carbon & $\mathrm{y}=2771.5 \mathrm{x}-79$ & $0.84 * * *$ \\
$\beta-$ glucosidase & Hot water soluble carbohydrate & $\mathrm{y}=0.56 \mathrm{x}+16.4$ & $0.95 * * *$ \\
$\beta-$ glucosidase & pH & $\mathrm{y}=-85.7 \mathrm{x}+816.6$ & $0.72^{* * *}$ \\
$\beta-$ glucosidase & Soil organic carbon & $\mathrm{y}=136.1 \mathrm{x}-260.9$ & $0.86^{* * *}$ \\
\hline
\end{tabular}

labile pool of soil $\mathrm{C}$, may be sensitive to perturbation and stress in the soil-plant ecosystems (Doran and Parkins, 1994) and therefore, could be used as a sensitive indicator of soil quality. Water extractable carbon is usually considerably smaller than other labile pool. In this case, it constituted between $1-7 \%$ of the microbial biomass carbon pool. Water extractable carbon represents only a small fraction of SOC but determines soil microbial activity (Janzen et al., 1992). Like SOC, there was a direct relationship between straw incorporation and water extractable carbon. Soil carbohydrates, which comprise about $5-25 \%$ of soil organic matter, are readily degradable components of SOM and the major energy sources for microorganisms. Haynes et al. (1991) found that the fraction of soil carbohydrate extractable with hot water was more closely correlated with aggregate stability than soil organic matter content. In our experiment the elevated concentration of hot water extractable carbohydrate at $50 \mathrm{~mm}$ and the marked decline in the subsoil horizons under no-till and chisel ploughing was matched by a concomitant decline in concentration of SOC.

\section{CONCLUSION}

No-till and chisel ploughing with crop straw incorporation provide the best strategy to maintain or improve the long-term quality and productivity of temperate arable soils in the South West England. These cultivation methods promote surface accumulation of straw enabling sequestration of $\mathrm{C}$ in the surface soil horizons. For weakly structured soils, maintenance of organic matter is vitally important to allow continued use of soilconserving minimum tillage systems. Our results confirm that no-till and chisel ploughing maintained carbon in the surface soil horizons, but mouldboard ploughing distributed carbon more uniformly throughout the soil profile, particularly when straw was incorporated. In terms of soil quality assessment, the significant positive correlation between hot water soluble carbon and SOC, microbial biomass carbon, $\beta$-glucosidase and acid phosphatase enzymes indicates that hot water soluble carbon is a sensitive indicator of tillage and straw management effects in winter wheat systems.
Acknowledgements: S. Hazarika was in receipt of a Commonwealth Commission Scholarship while this work was being carried out. North Wyke Research is grant aided by Biotechnology and Biological Sciences Research Council (BBSRC).

\section{REFERENCES}

Ahl C., Joergensen R.G., Kandeler E., Meyer B., Woehler V. (1998) Microbial biomass and activity in silt and sand loams after longterm reduction in tillage using the 'Horsch' system, Soil Till. Res. 49, 93-104.

Benbi D.K., Brar J.S. (2009) A 25-year record of carbon sequestration and soil properties in intensive agriculture, Agron. Sustain. Dev. $29,257-265$.

Carter M.R. (2005) Long-term tillage effects on cool-season soybean in rotation with barley, soil properties, and carbon and nitrogen storage for fine sandy loams in the humid climate of Atlantic Canada, Soil Till. Res. 81, 109-120.

Dalal R.C., Henderson P.A., Glasby J.M. (1991) Organic matter and microbial biomass in a vertisol after 20 years of zero-tillage, Soil Biol. Biochem. 23, 435-441.

Deng S.P., Tabatabai M.A. (1997) Effect of tillage and residue management on enzyme activities in soils: III. Phosphatase and arylsulfatase, Biol. Fert. Soils 24, 141-146.

Doran J.W., Parkins T.B. (1994) Defining and assessing soil quality, in: Doran J.W., Coleman D.C., Bezdicek D.F., Stewart B.A. (Eds.), Defining Soil Quality for a Sustainable Environment, Soil Science Society of America, Madison, pp. 3-21.

Ellert B.H., Bettany J.R. (1995) Calculation of organic matter and nutrients stored in soils under contrasting management regimes, Can. J. Soil Sci. 75, 529-538.

Findlay D.C., Colborne G.J.N., Cope D.W., Harrod T.R., Hogan D.V., Staines S.J. (1984) Soils and their Use in South West England, Soil Survey of England and Wales, Harpenden.

Gil-Sotres F., Trasar-Cepeda C., Leiros M.C., Seoane S. (2005) Different approaches to evaluating soil quality using biochemical properties, Soil Biol. Biochem. 37, 877-887.

Gregorich E.G., Liang B.C., Drury C.F., MacKenzie A.F., McGill W.B. (2000) Elucidation of the source and turnover of water soluble and microbial biomass carbon in agricultural soils, Soil Biol. Biochem. 32, 581-587.

Haynes R.J., Swift R.S., Stephen R.C. (1991) Influence of mixed cropping rotations (pasture-arable) on organic matter content, water 
stable aggregation and clod porosity in a group of soils, Soil Till. Res. 19, 77-87.

He J., Kuhn N.J., Zhang X.M., Zhang X.R., Li H.W. (2009) Effects of 10 years of conservation tillage on soil properties and productivity in the farming-pastoral ecotone of Inner Mongolia, China, Soil Use Manage. 25, 201-209.

Janzen H.H., Campbell C.A., Brandt S.A., Lafond G.P., Townley-Smith L. (1992) Light fraction organic matter in soils from long-term crop rotations, Soil Sci. Soc. Am. J. 56, 1799-1806.

Kushwaha C.P., Tripathi S.K., Singh K.P. (2001) Soil organic matter and water-stable aggregate under different tillage and residue conditions in a tropical dryland agroecosystem, Appl. Soil Ecol. 16, 229-241.

Lal R. (2008) Soils and sustainable agriculture: a review, Agron. Sustain. Dev. 28, 57-64.

Lichtfouse E. (1997) Heterogeneous turnover of molecular organic substances from crop soils as revealed by $13 \mathrm{C}$ labeling at natural abundance with Zea mays, Naturwissenschaften 84, 22-23.

Lichtfouse E., Sappin-Didier V., Denaix L., Caria G., Metzger L., Amellal-Nassr N., Schmidt J. (2005) A 25-year record of polycyclic aromatic hydrocarbons in soils amended with sewage sludges, Environ. Chem. Lett. 3, 140-144.

Lobe I., Bol R., Ludwig B., Du Preez C.C., Amelung W. (2005) Savannaderived organic matter remaining in arable soils of the South African Highveld long-term mixed cropping: Evidence from C-13 and N-15 natural abundance, Soil Biol. Biochem. 37, 1898-1909.

Montemurro F., Convertini G., Maiorana M., Ferri D. (2007) Alternative sugar beet production using shallow tillage and municipal waste fertiliser, Agron. Sustain. Dev. 27, 129-137.

Rasmussen P.E., Collins H.P. (1991) Long-term impacts of tillage, fertilizer, and crop residue on soil organic matter in temperate semiarid regions, Adv. Agron. 45, 93-94.

Roberto A., Díaz R.A., Barbero N., Santanatoglia O.J., Blotta L. (1995) Soil organic carbon, microbial biomass and $\mathrm{CO}_{2}-\mathrm{C}$ production from three tillage systems, Soil Till. Res. 33, 17-28.
Safarik I., Santruckova H. (1992) Direct determination of total soil carbohydrate content, Plant Soil 143, 109-114.

Scopel E., Findeling A., Guerra E.C., Corbeels M. (2005) Impact of direct sowing mulch-based cropping systems on soil carbon, soil erosion and maize yield, Agron. Sustain. Dev. 25, 425-432.

Smith P., Powlson D.S., Glendining M.J., Smith J.U. (1998) Preliminary estimates of the potential for carbon mitigating in European soils through no-till farming, Global Change Biol. 4, 679-685.

Sparling G.P. (1992) Ratio of microbial biomass carbon to soil organic carbon as a sensitive indicator of changes in soil organic matter, Aust. J. Soil Res. 30, 195-207.

Tabatabai M.A. (1994) Soil Enzymes, in: Weaver R.W., Angle J.S., Bottomley P.S. (Eds.), Methods of Soil Analysis, Part. 2. Microbiological and Biochemical Properties. SSSA book series No. 5, Soil Science Society of America, Madison, Wisconsin, USA, pp. $775-883$.

Vanden Bygaart A.J., Gregorich E.G., Angers D.A. (2003) Influence of agricultural management on soil organic carbon: a compendium and assessment of Canadian studies, Can. J. Soil Sci. 83, 363-380.

Wanniarachchi S.D., Voroney R.P., Vyn T.J., Beyaert R.P., MacKenzie A.F. (1999) Tillage effects on the dynamics of total and cornresidue-derived soil organic matter in two southern Ontario soils, Can. J. Soil Sci. 79, 473-480.

West T.O., Post W.M. (2002) Soil organic carbon sequestration rates by tillage and crop rotation: a global data analysis, Soil Sci. Soc. Am. J. 66, 1930-1946.

Wu J., Joergensen R.G., Pommering B., Chaussod R., Brookes P.C. (1990) Measurement of soil microbial biomass C by fumigation extraction - an automated procedure, Soil Biol. Biochem. 22, 11671169.

Zuazo V.H.D., Pleguezuelo C.R.R. (2008) Soil-erosion and runoff prevention by plant covers. A review, Agron. Sustain. Dev. 28, 65-86. 